\title{
RAPID EXTRACTION OF LANDSLIDE AND SPATIAL DISTRIBUTION ANALYSIS AFTER JIUZHAIGOU Ms7.0 EARTHQUAKE BASED ON UAV IMAGES
}

\author{
Q. S. Jiao*, Y. Luo, W. H. Shen, Q. Li, X. Wang \\ ${ }^{1}$ Institute of Crustal Dynamics, China Earthquake Administration, China - 1985jqs@163.com
}

Commission ICWG III/IVa

KEY WORDS: Jiuzhaigou earthquake, UAV, object-oriented, landslide extraction, spatial distribution analysis

\begin{abstract}
:
Jiuzhaigou earthquake led to the collapse of the mountains and formed lots of landslides in Jiuzhaigou scenic spot and surrounding roads which caused road blockage and serious ecological damage. Due to the urgency of the rescue, the authors carried unmanned aerial vehicle (UAV) and entered the disaster area as early as August 9 to obtain the aerial images near the epicenter. On the basis of summarizing the earthquake landslides characteristics in aerial images, by using the object-oriented analysis method, landslides image objects were obtained by multi-scale segmentation, and the feature rule set of each level was automatically built by SEaTH (Separability and Thresholds) algorithm to realize the rapid landslide extraction. Compared with visual interpretation, object-oriented automatic landslides extraction method achieved an accuracy of $94.3 \%$. The spatial distribution of the earthquake landslide had a significant positive correlation with slope and relief and had a negative correlation with the roughness, but no obvious correlation with the aspect. The relationship between the landslide and the aspect was not found and the probable reason may be that the distance between the study area and the seismogenic fault was too far away. This work provided technical support for the earthquake field emergency, earthquake landslide prediction and disaster loss assessment.
\end{abstract}

\section{INTRODUCTION}

On August 8, 2017, a magnitude 7.0 earthquake occurred in Jiuzhaigou county, Sichuan province. The focal depth was 20 $\mathrm{km}$. The earthquake caused great casualties and property losses. As of August 13, the earthquake caused 25 deaths and over 73,000 houses were damaged. The Zhangzha town is one of the most severely damaged areas by the earthquake. The earthquake caused the mountain break up and induced a large number of earthquake landslides. This type of disaster had a wide range of distribution and strong destruction, especially causing road blocking. Moreover, it caused the field investigation and earthquake rescue fail to carry out normally, which seriously affected the earthquake emergency investigation and disaster assessment. After the earthquake, related agencies used the remote sensing images to conduct landslides and road damage surveys, and interpreted the disasters distribution around the Zhangzha town near the epicenter.

Traditional landslide interpretation using remote sensing images was mainly based on visual interpretation. However its accuracy was high, it required much time and effort that cannot meet the timeliness needs of earthquake emergency and rapid assessment. To solve this problem, domestic and foreign scholars had carried out research on the automatic recognition and interpretation of landslides on remote sensing images. Fu (2006) used support vector machine method to automatically identify the landslide on Landsat TM images. Hua (2008) used unsupervised and supervised classification methods to achieve automatic extraction of landslide bodies based on Landsat TM images combined with different remote sensing indices. When remote sensing images of the earthquake zone in different time phases can be acquired after the earthquake, the landslides can be identified by using the change detection method. The methods above were mainly based on remote sensing images with

\footnotetext{
* Corresponding author
}

low and medium resolution. The processing units were mainly pixels which brought about the limitation of information recognition mode. And it hindered the objects correlation at different scales and led to the obvious salt and pepper effect of the results.

In view of the problems existing in the landslides identification on medium and low resolution images, by using the high spatial resolution UAV (unmanned aerial vehicle) images, the objectoriented method emerged and was widely used in the landslides identification. This method used the homogenous image object generated after the image segmentation as the basic analysis units, and comprehensively employed the spectral, texture, shape, and context information of the speckles to classify the image and realize the automatic information identification. Martin (2005) used object-oriented method to identify the soil and rock landslides on the Landsat ETM+ images and the overall accuracy was up to $65 \%$. Trigila (2010) used objectoriented method to extract the landslides of Aosta valley in northern Italy. Chen (2017) used object-oriented methods to realize the suspected landslides extraction based on the DEM and DOM images acquired by UAV. And some scholars had also combined object-oriented and change detection methods to extract earthquake damage information. Hu (2008) performed multi-scale segmentation of IKONOS and SPOT 5 images at different time phases to obtain image objects and then an objectoriented change detection method was used to extract landslides. Compared with traditional pixel-based extraction methods, the results were more accurate and realistic. Ren (2009) used object-oriented analysis building feature rule sets to classify images, and then used post-classification change detection methods to extract roads earthquake damage information based on the FORMOSAT and IKONOS images at different time phases. Ping (2011) used remote sensing images with different time phases to extract the landslides distribution in southern Italy with an accuracy of $81.8 \%$. The relevant research at home and abroad had proved that the object-oriented method can maintain the completeness of the image speckle on highresolution images, and the classification accuracy was 
significantly improved. This method not only depended on the image spectral, but also comprehensively considered the spatial structure and texture of the images objects to improve the identification accuracy. However, in the process of setting the segmentation parameters and the classification rules, the parameters needed to be continuously adjusted relying on the experiences with large time consuming. In the earthquake emergency response, it was necessary to take the classification speed and accuracy into account to realize the automatic landslides identification.

In order to improve the timeliness of remote sensing earthquake emergency, this paper aimed to extract landslides distribution quickly and accurately. On the basis of in-depth analysis of landslide image characteristics, the image feature sets of landslides were established. By using object-oriented analysis method based on UAV data the automatic construction algorithm of image feature rule sets were studied to build the landslides identification model in a short period after earthquake. This can improve the landslide identification efficiency, and break through the work mode of visual interpretation. At the same time, the spatial distribution characteristics of landslides were analyzed to provide information for landslide prediction and hazard investigation after the disaster.

\section{STUDY AREA AND DATA SOURCE}

According to the data issued by the China Earthquake Administration, the epicenter of the Jiuzhaigou earthquake was located near the Minjiang, Tazang, and Huya faults. Xu (2017) from the Institute of Geology, China Earthquake Administration thought that the seismogenic faults was the junction between the southern section of the Tazang fault and the northern section of the Huya fault. The active faults distribution near the epicenter was shown in Figure 1.

The satellite remote sensing image obtained after earthquake was greatly affected by the cloud, and the identification of the ground objects was poor Due to the urgency of the rescue, the authors carried UAVs to enter the disaster area as early as August 9 to obtain the aerial images near the epicenter. UAVs have the advantages of strong flexibility with high resolution image acquisition capability, and have been widely applied in the fields of geological environment and disaster investigation, post-earthquake emergency and disaster assessment. We conducted UAV aerial survey of Zhangzha town which was the nearest settlement to the epicenter and acquired $11.37 \mathrm{~km} 2$ images with spatial resolution of $0.2 \mathrm{~m}$. Figure 2 showed the image before and after the earthquake.

\section{IMAGE FEATURES OF THE LANDSLIDES}

Affected by strong ground motions and topography, the slopes were loosened or even disintegrated, resulting in collapsing and slipping. This was the general process of most seismic secondary disaster triggered by strong earthquakes. Rapid sliding was also an important feature of the seismic secondary landslides. Huang (2009) classified the Wenchuan earthquake landslides into five types: slipping type, collapse type, ejection type, stripping type, and shatter type according to the instability mechanism of the slope. Under the action of strong vibrations, seismic waves caused cracks in the slopes during the propagation process. Subsequently, under the action of strong ground motions, the slope collapsed along a specific "face". Comparing to the arc-shaped landslides formed in normal gravity conditions, the slipping landslides formed by strong ground motions were mainly based on the "late edge cracked surface". It was difficult for the slope damaged by the crack to be stable on the gliding bed and would overall pour down and collapse. If the front edge was not blocked, high-speed and long-distance sliding would occur. Therefore, the deposits formed by the earthquake landslides were usually far away from the slide bed and accumulated in the front of the slope foot.

Landslides could be identified on remote sensing images by features such as morphology, hue, texture, and shadow. Earthquake landslides were usually destructive to surface vegetation especially in areas with high vegetation coverage. Vegetation was severely damaged and the underlying rock and soil was bare. As a result, the image showed light tones and obvious texture features. The ground was also rough, as shown in Figure 3a. In terms of morphological characteristics, landslides showed as tongue, ellipse, pear-shaped, bench-shaped, horn-shaped, leaf-shaped, diamond-shaped, tile-shaped, or irregular shape in high-resolution remote sensing images. The side wall appeared a circle chair shape or "double groove homologous" micro-topography, as shown in Figure 3b. The earthquake landslides deposits slid forward and often covered roads and buildings which caused road jams, damming lakes, and the buildings destruction, as shown in Figure 3c.

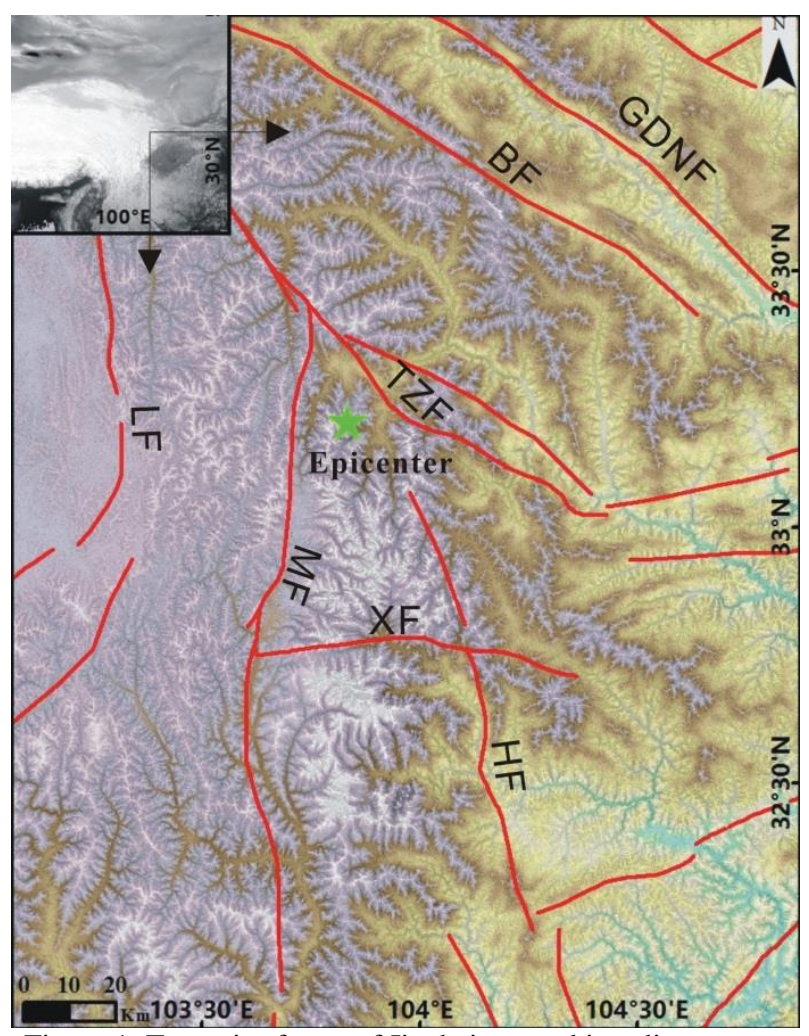

Figure 1. Tectonics frame of Jiuzhaigou and its adjacent area (Dai L X et al, 2017)

(The green star denoted epicenter. The red lines denoted active faults. GDNF: Guanggaishan-Dieshan Northern Fault; BF: Bailongjiang Fault; TZF: Tazang Fault; LF: Longriba Fault; MF: Minjiang Fault; XF: Xueshanliangzi Fault; HF: Huya Fault)

\section{METHODS}

In order to improve the timeliness of the disaster information acquisition, it was necessary to construct a quick and accurate method for identifying the disaster, and quickly extracted the disaster information after acquiring the data. The method proposed in this paper was to use the multi-scale segmentation method to segment the image and build the image object. Then, 
the automatic feature rule sets build method was used to extract the landslide information. The overall technical flow was shown in Figure 4.

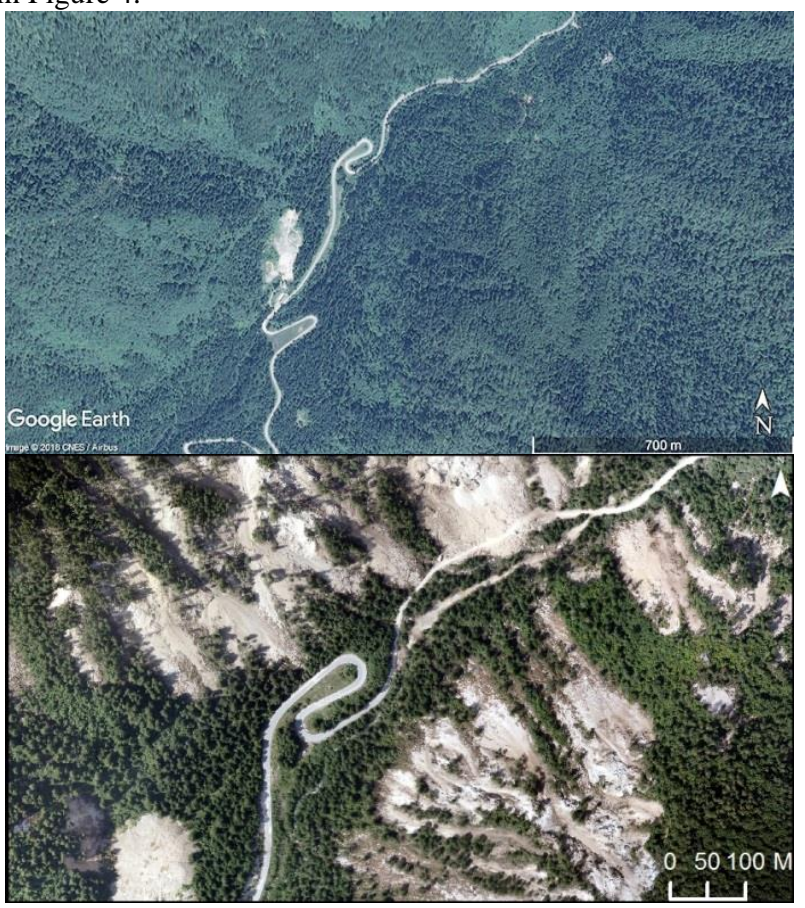

Figure 2. Comparison images of the study area pre- and postearthquake (The top map was Google Earth image before earthquake. The bottom map was UAV image)

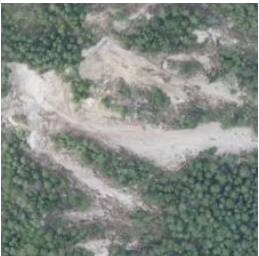

a

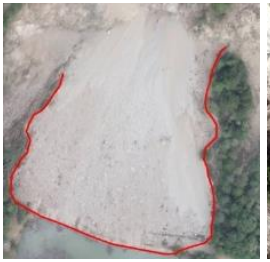

b

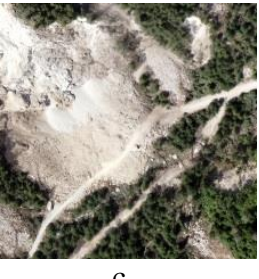

c
Figure 3. Aerial images of earthquake landslides

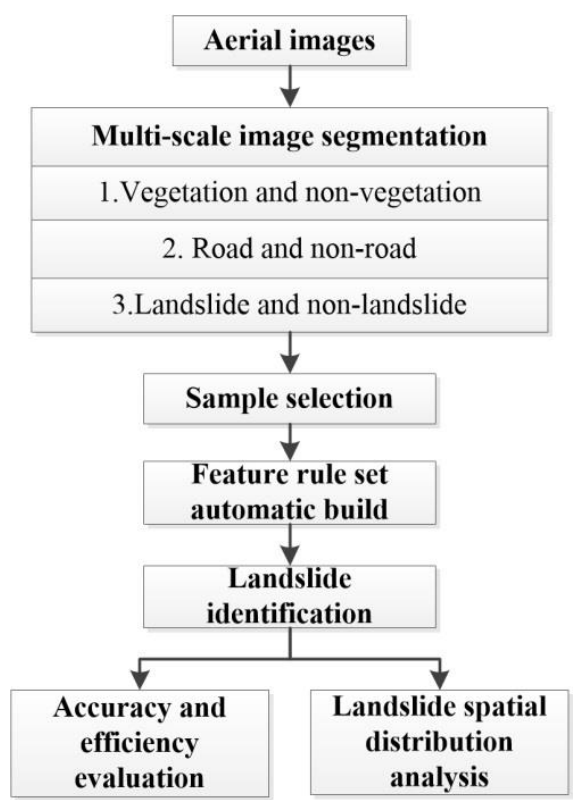

Figure 4. Technology flow chart of landslide identification and spatial distribution analysis based on object oriented method

\subsection{Image objects construction}

The image object was composed of multiple pixels with the same shape, spectrum, texture, and context features. The internal objects had the greatest homogeneity, and the external objects had the greatest heterogeneity. The types of ground features covered by high-resolution images acquired after earthquakes were various, and the scale of the ground objects was different. A single segmentation scale cannot completely identify landslide information. The multi-scale segmentation fully considered the multi-level of the objects physical object. The hierarchical network structure of different scales was used to reveal the size and hierarchical relationship of ground objects that solved the problems of fixed data source and the limitation of single-scale target recognition. At the same time, the trend of high-resolution image segmentation was to make full use of images features and the segmented image objects were closer to the result of visual interpretation. The multi-scale segmentation used the regional growth and merging algorithm based on the principle of minimum heterogeneity. The average heterogeneity of objects was synthetically expressed by the color (spectral) heterogeneity standard and the shape (space) heterogeneity standard. The average heterogeneity of the image objects was calculated from spectral heterogeneity parameters hcolor and shape heterogeneity parameters $h_{\text {shape }}$ (Baatz, 1999). The formula was as follows.

$$
f=w_{\text {color }} \cdot h_{\text {color }}+w_{\text {shape }} \cdot h_{\text {shape }}
$$

In the formula, wcolor was a custom color weight with data range $0 \leqslant w_{\text {color }} \leqslant 1$. The sum of $w_{\text {color }}$ and $w_{\text {shape }}$ was 1 . Shape heterogeneity was calculated from smoothness and compactness. The formula was as follows.

$$
h_{\text {shape }}=w_{\text {cmpct }} \cdot h_{\text {cmpct }}+\left(1-w_{\text {cmpct }}\right) \cdot h_{\text {smooth }}
$$

In the formula, wcmpct was the custom weight of the compactness with data range $0 \leqslant \mathrm{wcmpct} \leqslant 1$. In this experiment, the value of wcolor was 0.65 and the value of wcmpct was 0.5 .

For different segmentation thresholds, there were differences in the size of the object formed by the original pixel aggregation, and the setting of the segmentation threshold scale parameters also affected the classification effect. Therefore, it was necessary to select the optimal segmentation scale. For the earthquake landslides in the image, the ideal segmentation effect was that one or more image objects obtained after the segmentation clearly showed the boundary of the ground features, the segmented objects cannot be too broken, and the boundary cannot too ambiguous. Inappropriate segmentation thresholds would cause "insufficient segmentation" or "over segmentation".

Through image visual interpretation, it was found that the types of ground features mainly included vegetation, water, and landslides. Therefore, three-layer segmentation scale was set to describe the image object. The optimal segmentation threshold was manually set as 420,375 and 230 respectively.

\subsection{Feature rule set description}

The construction efficiency and completeness of feature rule set determined the efficiency and precision of the follow-up landslides extraction. The construction methods of feature rule sets mainly included visual interpretation and feature selection based on training samples. The visual interpretation method relied on human experience and required multiple attempts to select appropriate features and thresholds. The selection process was time-consuming and the selected features were usually incomplete. The feature rule set based on training samples was constructed by selecting the features of several image objects to 
obtain suitable features and thresholds. The method used in this paper was automatic features selection based on the training samples. Since the identified targets were mainly landslides, the SEaTH (Seperability and Thresholds) classification rules were used to automatically construct feature rule set in this experiment. Based on the derived sample feature values, this method realized the selection of the best features among different classes and the automatic determination of thresholds that automatically constructed eature rule set and improved efficiency.

The SEaTH method was first proposed by Nussbaum (2006) and could be used to solve the problem of optimal selection among massive features and automatic determination of significant feature thresholds in the process of object-oriented analysis. The SEaTH method consisted of two parts. The first part was the optimal feature selection, and the second part was the determination of the significant feature threshold.

In the first segmentation scale, spectral and texture features were selected as the main features, and the feature rule set for vegetation and non-vegetation identification was built by using SEATaTH. On the second segmentation scale, through the combination of spectral and shape features, the feature rule set for water and non-water identification was constructed. Finally, in the third segmentation scale, the integrated spectral, shape, and texture features were used to build feature rules set for the landslides identification. The automatically determined feature rule sets were shown in Table 1 .

\begin{tabular}{|c|c|c|}
\hline Level & Type & Feature rule set \\
\hline 1 & $\begin{array}{c}\text { vegetation } \\
\text { non-vegetation }\end{array}$ & $\begin{array}{c}\text { Homogenity }<=0.32 \text { and } 30<\text { Band } B<146 \\
\text { Homogenity }>0.32 \text { or } 30<=\text { Band B or } \\
\text { Band B }>=146\end{array}$ \\
\hline 2 & $\begin{array}{c}\text { water } \\
\text { non-water }\end{array}$ & $\begin{array}{c}110<\text { Band } \mathrm{G}<186 \text { and } 661<\text { Area }<9207 \\
\text { Band } \mathrm{G}<=110 \text { or Band } \mathrm{G}>=186 \text { or }\end{array}$ \\
\hline 3 & $\begin{array}{l}\text { landslide } \\
\text { non-landslide }\end{array}$ & $\begin{array}{c}\text { Brightness }>173.5 \text { and Entory }<0.96 \text { and } \\
\text { Second Moment }>0.76 \text { and Elliptic fit }<0.44 \\
\text { Brightness }<=173.5 \text { or Entory }<=0.96 \text { or } \\
\text { Second Moment }<=0.76 \text { or Elliptic fit }>= \\
0.44\end{array}$ \\
\hline
\end{tabular}

Table 1 Feature rule sets of different objects at different levels

\section{RESULTS}

The results of earthquake landslides extraction were shown in Figure 5. Most of the landslides were distributed along main road and 3large landslides block road S301 which brought great difficulties to the emergency rescue. A total of 58 landslides are extracted with a total area of $1.735 \mathrm{~km}^{2}$. The minimum landslide area was $20 \mathrm{~m}^{2}$. Landslide debris partially buried the road and caused road blockage.

\subsection{Accuracy evaluation}

In order to quantitatively describe the accuracy of landslides identification, the same aerial photos were used to do landslides visual interpretation. Then the user's accuracy (UA), producer's accuracy (PA), overall accuracy (OA), and Kappa coefficient were calculated by using the confusion matrix to measure the identification accuracy. The results were shown in Table 2. By using the object-oriented automatic extraction method, the overall accuracy of the landslide identification was $94.8 \%$. Compared with the visual interpretation result, due to the landslide debris rushed on the road surface, some roads were mistakenly classified as landslides.

\begin{tabular}{|c|c|c|c|c|}
\hline Type & PA & UA & OA & Kappa \\
\hline Landslide & $98.5 \%$ & $95.4 \%$ & $94.8 \%$ & 0.827 \\
\hline
\end{tabular}

Table 2 Accuracy analysis of landslide identification results

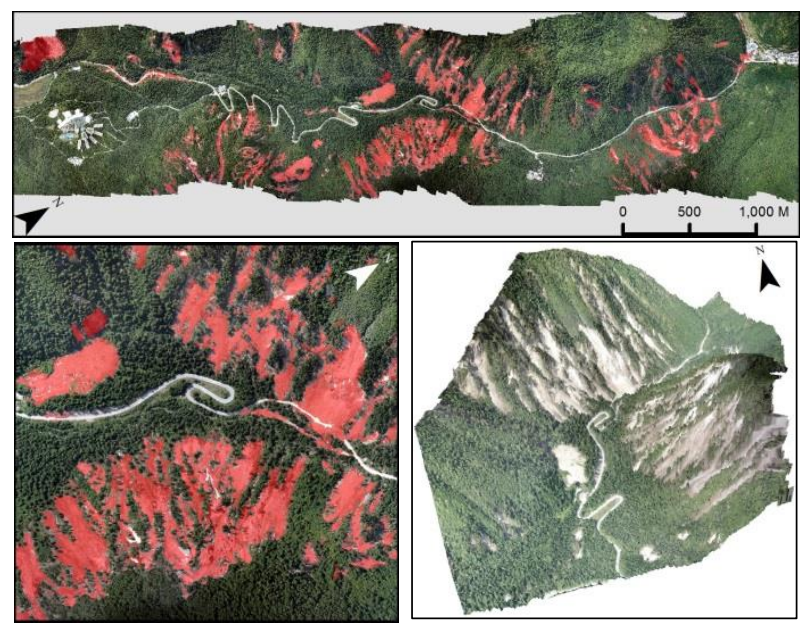

Figure 5. Automatic extraction result of earthquake landslides

\subsection{Efficiency evaluation}

During the earthquake emergency, the rapid disaster identification and evaluation was very important for emergency rescue. In order to test the efficiency, the automatic classification and visual interpretation were carried out at the same time. The interpreter had rich experience and software operation basis. The object-oriented automatic classification method had higher efficiency under the same computer configuration. The object-oriented method only took half the time compared with visual interpretation. With the computational performance improved, the automatic disaster information extraction would gradually develop into the main method for earthquake emergency and disaster assessment.

\subsection{Landslide spatial distribution analysis}

The landslides spatial distribution had important reference and guiding significance for risk assessment of secondary disasters and disaster prevention. The earthquake landslides were caused by ground motions, and topography was also an important gestation factors. Statistical analysis of topographic factors could acquire the topographic features of landslides and then analyse their distribution patterns. In this paper, elevation, slope relief, aspect and roughness were selected as topographic factors to carry out statistical on the landslides spatial distribution. Each topographic factor was classified into five section using equal intervals. Then the landslide point and the classified topographic factors were overlaid and the spatial statistical analysis function was used to analyse the distribution regularities (Figure 6). It was found that the earthquake landslide was mainly distributed in slope of $39.8-69.8^{\circ}$, elevation of $2623 \mathrm{~m}-2935 \mathrm{~m}$, aspect of $14.8-44.3$, relief of 131 227 , and the roughness of 1.16-2.09. The spatial distribution of earthquake landslides was positively correlated with the slope and relief, negatively correlated with roughness, and had no obvious relationship with aspect and elevation. According to the statistical results of landslides in the Wenchuan earthquake, the landslides distribution was affected by the seismic wave propagation and the fault movement. It could be classified out into "back slope effect" and "directional effect of fault dislocation" (Xu, 2010). Earthquake landslides were controlled by active faults. The spatial distribution of landslides was related to the distance from active faults and could provide a 
certain proof for the identification of the seismogenic fault. In this experiment, the relationship between the landslide spatial distribution and the slope was not obvious. The possible reason may be that the research area was far from the origin seismogenic fault.

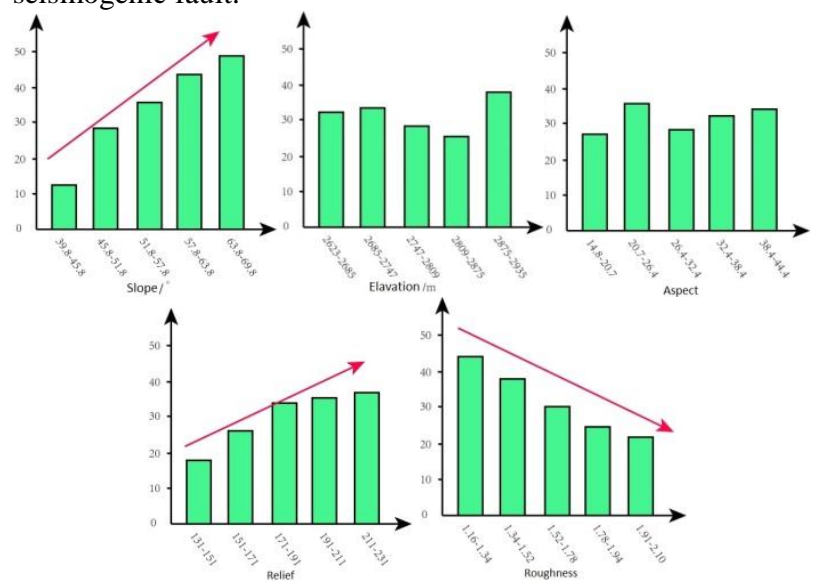

Figure 6. Spatial distribution of earthquake landslides under different topographic factors

The landslides spatial distribution was also affected by the lithology to a certain extent. Due to the high vegetation cover in the study area, lithology cannot be identified on images. The relationship between landslides spatial distribution and lithology were not been analysed. The analysis of earthquake landslides spatial distribution could provide information support for subsequent landslide risk assessment and prediction. The follow-up work should consider increasing landslide samples and bringing in more factors to further determine the influence factors on landslides distribution.

\section{CONCLUSIONS}

On the basis of summarizing the earthquake landslides characteristics in aerial images, by using the object-oriented analysis method, landslides image objects were obtained by multi-scale segmentation, and the feature rule set of each level was automatically built by SEaTH (Separability and Thresholds) algorithm to realize the rapid landslide extraction. Compared with visual interpretation, object-oriented automatic landslides extraction method achieved an accuracy of $94.3 \%$. The spatial distribution of the earthquake landslide had a significant positive correlation with slope and relief and had a negative correlation with the roughness, but no obvious correlation with the aspect. The relationship between the landslide and the aspect was not found and the probable reason may be that the distance between the study area and the seismogenic fault was too far away. This work provided technical support for the earthquake field emergency, earthquake landslide prediction and disaster loss assessment. In the follow-up work, we needed to study the automated segmentation algorithm to further improve the accuracy of information identification and really make this work play an important role in earthquake emergency.

\section{ACKNOWLEDGEMENTS}

This work was supported by a research grant from the Institute of Crustal Dynamics, China Earthquake Administration [grant number ZDJ2017-29]

\section{REFERENCES}

Baatz M, Schape A, 1999.Object-Oriented and Multi-Scale image Analysis in Semantic Networks. Proceedings of the second International Symposium on Operationalization of Remote Sensing, ITC, pp.2563-2575.

Baiz U C, P. Hofinaim, G. Wilihauck, L Lingenfelder, M. Heynen, 2004. Multi-resolution object-oriented fuzzy analysis of remote sensing data for GIS-ready information. ISPRS Journal of Photogrammetry and Remote Sensing, (58), pp. 239-258.

Blaschke T, Lang S, Hay G J, 2010.Object based image analysis for remote sensing. ISPRS journal of Photogrammetry and Remote Sensing, (1), pp. 2-16.

Chen T B, Hu Z W, Wei L, Hu S Q, 2017. Data processing and landslide information extraction based on UAV remote sensing. Journal of Geo-information Science, 19(5), pp. 692-701.

Dai L X, Xu Q, Fan X M, Chang M, Yang Q, Yang F, Ren J, 2017. A preliminary study on remote sensing interpertation of landslides triggered by Jiuzhaigou earthquake in Sichuan on August 8th, 2017 and their spatial distribution patterns. Journal of Engineering Geology, 25(9) , pp. 1151-1164.

Fu W J. Hong J Y, 2006. Discussion on application of support vector machine technique in extraction of information on landslide hazard from remote sensing images. Research of Soil and Water Conservation, 13(4), pp. 120-122

Hu D Y, Li J, Zhao W J, Peng G X, 2008. Object-oriented landslide detection from remote sensing imageries with high resolution. Journal of natural disasters, 17(6), pp. 42-46.

Hua L Z, Cui S H, Li X H, Yin K, Qiu Q Y, 2008. Remote sensing identification of earthquake trigged landslide and their impacts on ecosystem services: a case study of Wenchuan Country. Acta ecologica sinica, 33(3), pp. 96-301.

Huang R Q, 2009. Mechanism and geomachanical modes of landslide hazards triggered by Wenchuan 8.0 earthquake. Chinese journal of rock mechanics and engineering, 28(6), pp.1239-1249.

Li Y S, Huang C, Yi S J, Wu C H, 2017. Study on seismic fault and source rupture tectonic dynamic mechanism of Jiuzhaigou Ms7.0 earthquake. Journal of Engineering Geology, 25(4), pp.1141-1150.

Martin Y E, Frankli S. E, 2005. Classification of soil and bedrock-dominated landsides in British Columbia using segmentation of satellite imagery and DEM data. International journal of remote sensing, 26(7), pp. 1505-1509.

Nussbaum S, Niemeyer J, Canty M J, 2006. SEaTH-A New tool for automated feature extraction in the context of object-based image analysis for remote sensing. The 1st International Conference on Object-Based Image Analysis, Salzhourg,Austria.

Ping L A, Stumpf N, Kerle N, Casagli, 2011. Object-oriented change detection for landslide rapid mapping. IEEE geoscience and remote sensing letters, 8(4), pp.701-705.

Ren Y H, Liu Y L,Wei C J,Hu L Q, 2009.Study on information extraction method to road damage in Wenchuan earthquake 
The International Archives of the Photogrammetry, Remote Sensing and Spatial Information Sciences, Volume XLII-3, 2018 ISPRS TC III Mid-term Symposium "Developments, Technologies and Applications in Remote Sensing", 7-10 May, Beijing, China

based on high-resolution remote sensing images. Remote sensing technology and application, 24(1), pp.52-56.

Trigila A, Iadanza C, Spizzichino D, 2010.Quality assessment of the Italian landslide inventory using GIS processing. Landslides, 7(4), pp. 455-470.

Xu Q, Li W L, 2010. Study on the direction effects of landslides triggered by Wenchuan earthquake. Journal of Sichuan University (Engineering Science Edition), (S1), pp.7-14.

Xu X W, 2017. Seismotectonic map of Jiuzhaigou MS7.0 Earthquake. http://www.csi.ac.cn/manage/eqDown/05LargeEQ/201708082119M7. 0/zonghe.html 